\title{
Antigen sensing via nanobody-coated transistors
}

\author{
Organic electrochemical transistors functionalized with antigen-specific nanobodies can rapidly and specifically \\ detect antigens at attomolar-to-nanomolar levels in bodily fluids.
}

\section{Howard E. Katz}

$\Lambda$ ssays based on chemical interactions - most notably, the double-binding 'sandwich' enzyme-linked immunosorbent assay (ELISA) - are commonplace in research and clinical laboratories, yet they require specialist training and are slow. Electronic biosensors provide faster responses - on the timescale of seconds ${ }^{1}$ - and do not typically require laborious analytical processes, such as multiple rinsing steps. For electronic biosensors to detect the presence of a specific biomolecule in solution, the biomolecule must selectively bind to the biosensor. When biomolecular analytes of interest (typically nucleic acids, carbohydrates or proteins) bind to the active surface of the biosensor, they change its local or interfacial potential, or the impedance of the circuit that incorporates the binding site. These changes occur because of ion or dipole-moment rearrangements, or because of changes in the density or connectivity of molecular subunits on the biosensor. The changes in a biosensor's electronic properties occurring upon analyte binding can then be amplified via field-effect transistors ${ }^{2-5}$ (FETs; Fig. 1a). In fact, by means of a graphene electrode coated with specific antibodies for the spike protein of severe acute respiratory syndrome coronavirus-2 (SARS-CoV-2), FET-based biosensors could be used to detect coronavirus disease 2019 (COVID-19) ${ }^{6}$. However, complex device designs, fabrication difficulties associated, in particular, with antibody immobilization, and less competitive analytical performance (in particular regarding the sensitivity, selectivity and stability of the assay) have precluded the wider adoption of electronic biosensors. Reporting in Nature Biomedical Engineering, Sahika Inal, Raik Grünberg, Stefan Arold and colleagues now show that organic electrochemical transistors (OECTs) functionalized with antigen-specific nanobodies can detect the spike proteins from SARS-CoV-2 and Middle East respiratory syndrome coronavirus (MERS-CoV) within 10 minutes, at the single-molecule level, and in saliva, serum and nasopharyngeal samples? .

a

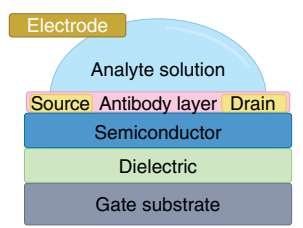

b

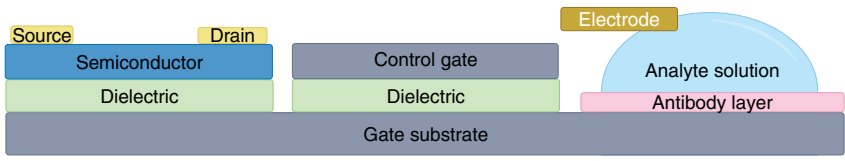

C
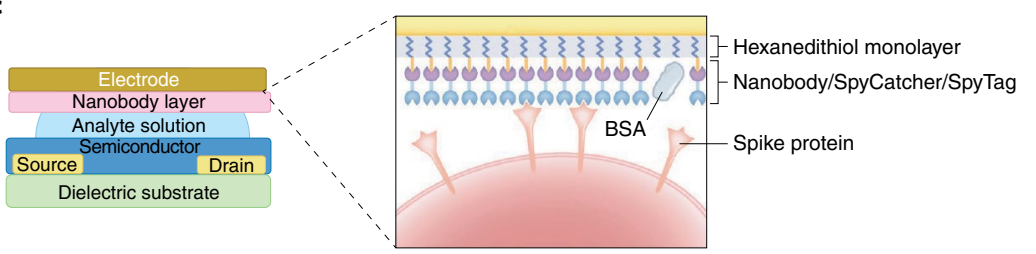

Fig. 1 | Biosensors based on FETs. a, In a traditional FET-based biosensor, the binding of the analyte to the antibody layer modifies the capacitive charge (induced by the substrate, which acts as a gate) in the semiconductor, across which the current from the source electrode to the drain electrode is measured. b, In an alternative configuration, the analyte solution can be separated from the semiconductor. The current is perturbed via the binding of the analyte to the antibody layer, which modifies the capacitive coupling of the substrate (which acts as a gate) with the semiconductor (through the dielectric). c, In Inal and colleagues' OECT, the capacitive and ionically compensated charge in the semiconductor is modified by the binding of the analyte to the electrode, which acts as a gate. Inset: the binding of the analyte (here, the SARS-CoV-2 virus) to the gold electrode involves a self-assembled chemical monolayer (dithiols linking the electrode to the SpyTag peptide) and a self-assembled oriented biological monolayer (of nanobodies for the virus's spike protein fused to the SpyCatcher protein). The addition of bovine serum albumin (BSA) prevents unspecific binding. Inset in c reproduced with permission from ref. ${ }^{7}$, Springer Nature Ltd.

Inal and co-authors' device consists of a polymer semiconductor coupled with a nanobody-functionalized gate electrode via the analyte solution (Fig. 1c). Changes in the voltage applied to the gate electrode are measured as downstream changes in the semiconductor's drain current (at a fixed drain voltage), and the current changes per unit of gate voltage were up to three orders of magnitude higher than in previously reported OECT devices ${ }^{8-10}$. Notably, because the semiconductor is not functionalized, the current-carrying part of the OECT is reusable (that is, only the nanobody-functionalized gates need to be changed). As is typical for electronic biosensors, the speed of detection was limited by the sample-introduction step and by molecular-transport processes rather than by the electronic-response time. Traditionally, in FET-based biosensors the binding of the analyte occurs on the surface of the semiconductor. However, it has become clear that separating the analyte-binding surface from the current-carrying components to which they are capacitively coupled increases device stability and signal-to-noise ratios ${ }^{1,8,11-14}$ (Fig. 1b). In such configurations, the semiconductor is not subject to leakage currents originating in the solution ${ }^{15}$.

Rather than using full antibodies (consisting of two heavy chains and two light chains), as is typical for biosensing FETs, Inal and co-authors functionalized the OECT gates with camelid-derived 
nanobodies (heavy-chain-only antibodies derived from the Camelidae family of mammals). The spike-specific nanobodies were expressed along with a linking 'fusion' protein (the SpyCatcher protein) conjugated with a complementary peptide (SpyTag; the SpyCatcher-SpyTag conjugate has been widely used to immobilize enzymes on surfaces), and attached to a functionalized alkylthiol layer on gold. The full nanobody-layer assembly was highly oriented and densely packed, which should promote the selectivity and sensitivity of protein detection. Moreover, the authors used polyether-substituted polythiophene to decrease the power needed for biomolecular detection and to increase detection sensitivity and expand its dynamic range, possibly due to enhanced gate-induced ion penetration into the conducting pathway. However, it is unclear why the optimized area of the gate electrode was 640 times larger than the active area of the semiconductor; a circuit model that accounts for this optimized arrangement may provide insight into, for example, whether such a large gate-to-semiconductor area ratio assists signal amplification by decreasing the total number of charges that must be introduced into the semiconductor to reach a desired change in conductance.

In fact, when functionalized with a nanobody for the green fluorescent protein, the biosensor's response arose from increased impedance at the gate electrode, decreasing the effective gate voltage applied to the semiconductor. This would be consistent with just a few bound proteins obstructing a substantial fraction of the 'macroscopic' area of the gate and thus blocking parallel paths from the gate electrode (from areas without bound protein) to the analyte solution. The absence of changes in the gate current suggests that such pathways are not blocked by the bound proteins (unless the limiting impedance is at the semiconductor interface), making the impedance change entirely capacitive (rather than conductive) and in series (rather than in parallel) with the conductive paths. An alternative explanation for the electronic effects at the gate could be that protein binding may change the interfacial potential, which would not be ruled out by unblocked conductive paths if binding caused ionic reorganization that extended over a device area much larger than the area of the bound protein.

Detecting the SARS-CoV-2 spike protein with high specificity (the biosensor distinguished the closely related spike proteins from SARS-CoV-2 and MERS-CoV) and at attomolar-to-nanomolar levels in saliva is particularly promising. However, as Inal and co-authors note, the practical implementation of the biosensor will most likely require integration with a microfluidic setup to streamline the operation of the biosensor (in particular, sample handling) and to decrease variabilities in sample volumes, in mixing and in travel times to the active electronic surfaces. The COVID-19 pandemic has added urgency to the development of fast and inexpensive assays that can determine the levels of virus-associated antigens in bodily fluids. In this regard, if the electronic biosensor can be developed for point-of-care use, the performance benefits over ELISA-based assays would be evident.

\section{Howard E. Katz (D) $₫$}

Department of Materials Science and Engineering, Johns Hopkins University, Baltimore, MD, USA.

$\varpi_{e-m a i l: h e k a t z @ j h u . e d u}$

Published online: 16 July 2021

https://doi.org/10.1038/s41551-021-00765-2

\section{References}

1. Song, J. et al. Adv. Funct. Mater. 27, 1606506 (2017).

2. Kergoat, L., Piro, B., Berggren, M., Horowitz, G. \& Pham, M. C. Anal. Bioanal. Chem. 402, 1813-1826 (2012).

3. Lin, P. \& Yan, F. Adv. Mater. 24, 34-51 (2012).

4. Torsi, L., Magliulo, M., Manoli, K. \& Palazzo, G. Chem. Soc. Rev. 42, 8612-8628 (2013).

5. Zhang, G. J. \& Ning, Y. Anal. Chim. Acta 749, 1-15 (2012).

6. Seo, G. et al. ACS Nano 14, 5135-5142 (2020).

7. Guo, K. et al. Nat. Biomed. Eng. https://doi.org/10.1038/s41551021-00734-9 (2021).

8. Macchia, E. et al. Mater. Horiz. 7, 999-1013 (2020).

9. Macchia, E. et al. Flex. Print. Electron. 3, 034002 (2018).

10. Inal, S., Malliaras, G. G. \& Rivnay, J. Nat. Commun. 8, 1767 (2017).

11. Barbaro, M. et al. IEEE Electron. Dev. Lett. 27, 595-597 (2006).

12. Demelas, M. et al. J. Mater. Chem. 1, 3811-3819 (2013).

13. Holzer, B. et al. Adv. Biosys. 1, 1700055 (2017).

14. Picca, R. A. et al. Adv. Funct. Mater. 30, 1904513 (2020).

15. Picca, R. A. et al. Front. Chem. 7, 667 (2019).

\section{Competing interests}

The author declares no competing interests. 